\title{
Onwards and upwards
}

\author{
Investment in large-scale science facilities is vulnerable in the present economic climate. Although some \\ projects must end, and others are under threat, there is success to be enjoyed in 2011 , and beyond.
}

In early January, the closure of Fermilab's Tevatron accelerator in 2011 was confirmed. Last year, physicists working on the two Tevatron experiments, CDF and D0, had won the backing of the US Department of Energy's High Energy Particle Advisory Panel to extend the accelerator's run for another three years, through to late 2014. That support was, however, contingent on the necessary funding - US\$35 million each year being injected and not stripped from other areas of the science budget. The Office of Science has now decided that the additional money cannot be found and the Tevatron must close as originally planned later this year.

There is of course palpable disappointment at the decision. The Tevatron has a long history, having come into operation in 1985, and having generated the proton-antiproton collisions in which, in 1995, the top quark was discovered. But the accelerator has come into its own in recent years, delivering ever increasing amounts of data that have made it possible for CDF and D0 physicists to push harder than ever against the bounds of the standard model. The model remains intact; there is no sign of the Higgs boson and, despite some interesting hints (discrepancies between theory and data at around the three-sigma level), no other new physics either.

Three more years of Tevatron running would have allowed those 'hints' to be chased down, and would certainly have told us more about the standard-model Higgs boson - although more likely where it's not (by setting 95\%-confidence-level exclusions across a wide range of mass values), than where it is. There is always more to be learned from more data, and any scientist would be reluctant to throw the switch on apparatus that now performs as well as the Tevatron and the CDF and D0 detectors do. But it is sensible that the Tevatron now make way for the new kid on the block, CERN's Large Hadron Collider (LHC), which has taken over as the world's highest-energy accelerator.

Director of Fermilab Pier Oddone must now chart the way ahead for the laboratory. The Tevatron, running at the energy frontier, was its signature programme, but Fermilab's accelerator facilities are already integral to various projects, present and future, that exploit instead the sheer intensity of its particle beams, particularly for the study of neutrino physics. It will be, says Oddone, "a complex transition for the laboratory" (see Director's Corner, Fermilab Today, 11 January 2011; http:// go.nature.com $/ 2 \mathrm{i} 7 \mathrm{cWc}$ ). For the moment, the situation remains "fluid", however, as budgets are not yet clear: the President's budget request for the financial year 2012 will be made this month.

\section{These are uncertain times and concern is rife for big science that requires big facilities.}

Another plea for additional funding has also recently gone unfulfilled, this time from the Deep Underground Science and Engineering Laboratory (DUSEL). The Homestake mine in South Dakota, USA, was famously the site of experiments by Ray Davis that established the solar neutrino problem in the late 1960s. Neutrino detection is a tricky business and the layers of rock above the mine caverns are an ideal shield against cosmic rays hence the proposal to create DUSEL there. Planned experiments include the Long Baseline Neutrino Experiment, in which a neutrino beam would be fired from Fermilab, 1,000 km away, to be detected at DUSEL; and LUX, to search for dark matter.

Safety concerns and groundwater issues as the mine is prepared have, however, led to a request for up to US $\$ 30$ million of additional funds ( $\$ 300$ million of the expected $\$ 800$ million total cost have already been committed). The denial of that request by the US National Science Board has left an uncertain situation, as DUSEL collaborators consider how preparatory work in the mine might continue and the final decision on the future of DUSEL, which had been expected this year from the National Science

Foundation, faces delay.

These are uncertain times and concern is rife for big science that requires big facilities. In the UK, for example, the research budget escaped the round of cuts last autumn (although, by remaining flat over four years, the budget will of course suffer a substantial real-terms cut), but capital expenditure took the hit: the money available to invest in the building and maintenance of laboratories and other science facilities was cut by more than $40 \%$.

Fermilab and DUSEL face challenging futures, and it is fortunate at this moment to be reminded of the rewards of dedicated development and long-term investment in science. After six years of drilling in the Antarctic ice, the IceCube experiment is now complete. The last of its detectors, which all together form an array of a cubic kilometre in volume, was lowered into the ice at the end of 2010. This remarkable, imaginative project will now tackle outstanding questions in neutrino physics and the mystery of ultra-high-energy cosmic rays.

Meanwhile, in early January, the first data from the European Space Agency's Planck satellite was presented at a conference in Paris, France. Planck's central result - a precise map of the cosmic microwave background radiation - is still to come (in early 2013) but the steps taken so far to understand both the performance of the apparatus and a host of other astronomical observables suggest success for this mission.

2011 should also be a good year for the LHC. As Nature Physics goes to press, CERN's accelerator physicists are meeting to decide the plan for the year. On the table, following a highly successful run through 2010, are the possibility of edging up the machine's energy, from 3.5 to $4 \mathrm{TeV}$ per beam, and of extending the run through 2012, which had originally been scheduled as a year of maintenance and upgrading. Both measures improve the chances of finding a Higgs boson sooner rather than later - and underline the sense in the decision, tough as it is, to call time on the Tevatron. 